$21 / 2$ year-old boy who developed acute paraparesis after a fall, a 17-year-old girl who developed hemianesthesia, nystagmus, dysarthria, and tongue deviation 1 week after chiropractic manipulations, and acquired torticollis after endotracheal anesthesia for tonsillectomy. All cases were found to have Chiari I malformation; only one had an associated syringomyelia. The diagnosis of Chiari I malformation hăs been facilitated by the use of MRI and asymptomatic cases are being uncovered by this imaging technique.

\title{
ATAXIA-OCULOMOTOR APRAXIA SYNDROME
}

A new spinocerebellar degenerative syndrome has been described in 14 patients from 10 families and reported from the Hopital des Enfants Malades, Paris, France; Montreal Neurological Institute, Canada; Rashid Hospital, Dubai, UAE; Tokyo Women's Medical College, Japan; and University of Colorado Medical School, Boulder, CO. Six of the cases had been reported previously in the Japanese literature and in abstracts. The clinical features included onset between 2 and 7 years of age, ataxia, ocular motor apraxia, choreoathetosis, depressed or absent deep tendon reflexes, dysarthria, masklike facies, intention tremor, and mildly subnormal intellectual function in one half the patients. СT was normal in 6 and showed mild vermal atrophy in 1 . None had conjunctival telangiectasia or abnormal immunoglobulins and a diagnosis of ataxia telangiectasia was considered unlikely. The syndrome was probably genetically determined with an autosomal mode of inheritance; it involved both sexes with consanguinity in 6 of 10 sibships. (Aicardi J, Barbosa C, Andermann E and F, Morcos R, Ghanem Q, Fukuyama Y, Awaya Y, Moe P. Ataxia - ocular motor apraxia; a syndrome mimicking ataxia - telangiectasia. Ann Neurol Oct $1988 ; \underline{24}: 497-502)$.

COMMENT. The differential diagnosis includes Cogan's ocular motor apraxia and ataxia telangiectasia. The authors, of whom 4 are already distinguished by eponymous syndromes (Aicardi $\mathrm{J}$, Andermann $\mathrm{E}$ and $\mathrm{F}$, and Fukuyama $\mathrm{Y}$ ), report a specific neurodegenerative syndrome of genetic origin with patients originating from widely separate geographical areas and from different ethnic backgrounds. Ataxia, areflexia, and ocular muscle paralyses are also featured in the Fisher syndrome.

\section{SEIZURES AND DIET}

\section{OLIGOANTIGENIC DIET FOR EPILEPSY AND MIGRAINE}

A diet low in antigenic items was used to treat 63 children with epilepsy refractory to medication at the Depts of Neurology, Immunology, and Dietetics, The Hospital for Sick Children, Great Ormond Street, and the Institute of Child Health, London, England. The authors had previously reported beneficial effects of the "oligoantigenic" diet in the treatment of migraine (Lancet $1983 ; \underline{2}: 865$ ) and the hyperkinetic syndrome (Lancet 1985; 1:940). The diet consisted of 2 meats (lamb and 
chicken), 2 carbohydrates (potatoes and rice), 2 fruits (banana and apple), vegetables (cabbage, sprouts, cauliflower, broccoli, cucumber, celery, carrots, parsnips), water, salt, pepper, pure herbs, and calcium and vitamins for 4 weeks. Patients who responded (no seizures or migraine for the last 2 weeks) were reintroduced to essential foods (eg. milk, cheese, wheat) at the rate of one a week. If symptoms were provoked, soy-based or goat milk products, rye or oats were substituted. Setbacks were avoided by first giving foods least likely to be antigenic (eg. beef, oats, peaches, or grapes). of 45 children who had epilepsy with recurrent headaches, abdominal symptoms, or hyperkinetic behavior, 25 had no seizures and 11 had fewer seizures during diet therapy. Foods most likely to provoke seizures when reintroduced were cow milk and cheese, citrus fruits, wheat, tartrazine and benzoic acid food additives, eggs, tomato, pork, and chocolate. In double-blind, placebocontrolled provocation studies introducing cow milk, orange juice, wheat, pork, egg, and benzoate, symptoms recurred in 15 of 16 children, including seizures in 8 ; none recurred with placebo. The oligoantigenic diet was unsuccessful in the treatment of 18 children who had epilepsy uncomplicated by migraine or hyperkinetic behavior. (Egger $\mathrm{J}$ et al. Oligoantigenic diet treatment of children with epilepsy and migraine. J Pediatr Jan 1989; $114: 51-58)$.

COMMENT. If reproducible and sustained, these results are impressive and deserve further investigation in children with frequently recurrent seizures and headache resistant to anticonvulsant medication. The authors point out that the diets are socially disruptive and may cause malnutrition. In the US, pediatric allergists are not generally impressed with the theory of food hypersensitivity as a cause of neurological disease and their enthusiastic collaboration in studies of this type is not readily available.

\section{VITAMIN E AND EPILEPSY}

The value of D-alpha-tocopheryl acetate (Vitamin E 400 IU/day) as an adjunct therapy for drug resistant epilepsy is reported from The Hospital for Sick Children and the University of Toronto Faculty of Medicine, Canada. In a randomized, double-blind, placebo-controlled trial, 10 of 12 children aged 6-17 years showed a greater than $60 \%$ reduction in seizure frequency whereas none in the control group showed a significant change. One-half of the responders had concomitant EEG improvements. The study period was 9 months: 3 mo pre-trial, 3 mo double-blind, and 3 mo open-label trial in which patients receiving placebo initially changed to Vitamin $\mathrm{E}$ as their own controls. The majority had generalized tonic-clonic seizures and anticonvulsant drug levels showed no significant change during treatment with Vitamin $E$. Plasma Vitamin $E$ levels increased from 5 to $37 \mathrm{mcM}$ during the treatment phase, the variability dependent on body size. Improvement in seizure control was similar in the open-label phase and no clinically significant alterations of blood counts, SGOT, alkaline 